\title{
Probing photoinduced spin states in spin-crossover molecules with neutron scattering
}

\author{
K. Ridier, ${ }^{1}$ G. A. Craig, ${ }^{2}$ F. Damay, ${ }^{1}$ T. Fennell,${ }^{3}$ M. Murrie, ${ }^{2}$ and G. Chaboussant ${ }^{1, *}$ \\ ${ }^{1}$ Laboratoire Léon Brillouin, UMR12 CEA-CNRS, F-91191 Gif-sur-Yvette, France \\ ${ }^{2}$ WestCHEM, School of Chemistry, University of Glasgow, G12 8QQ Glasgow, United Kingdom \\ ${ }^{3}$ Laboratory for Neutron Scattering, Paul Scherrer Institut, CH-5232 Villigen, Switzerland \\ (Received 14 July 2016; revised manuscript received 25 November 2016; published 3 March 2017)
}

\begin{abstract}
We report a neutron-scattering investigation of the spin-crossover compound $\left[\mathrm{Fe}(\mathrm{ptz})_{6}\right]\left(\mathrm{BF}_{4}\right)_{2}$, which undergoes an abrupt thermal spin transition from high spin (HS), $S=2$, to low spin (LS), $S=0$, around $135 \mathrm{~K}$. The HS magnetic state can be restored at low temperature under blue/green light irradiation. We have developed a specially designed optical setup for neutron scattering to address the magnetic properties of the light-induced HS state. By using neutron diffraction, we demonstrate that significant HS/LS ratios (of up to 60\%) can be obtained with this experimental setup on a sample volume considered large (400 $\mathrm{mg}$ ), while a complete recovery of the LS state is achieved using near-infrared light. Finally, with inelastic neutron scattering (INS) we have observed magnetic transitions arising from the photo-induced metastable HS $S=2$ state split by crystal-field and spin-orbit coupling. We interpret the INS data assuming a spin-only model with a zero-field splitting of the $S=2$ ground state. The obtained parameters are $D \approx-1.28 \pm 0.03 \mathrm{meV}$ and $|E| \approx 0.08 \pm 0.03 \mathrm{meV}$. The present results show that in situ magnetic inelastic neutron-scattering investigations on a broad range of photomagnetic materials are now possible.
\end{abstract}

DOI: 10.1103/PhysRevB.95.094403

\section{INTRODUCTION}

Molecular or nanometer-size systems capable of changing their magnetic state through some external stimulus (magnetic field, electric field, light, or pressure) are subject to intense studies. Promising examples are photoswitchable materials based on the spin transition mechanism for future applications as optical memories or digital displays [1]. The spin transition usually occurs with a temperature change, under pressure, or under visible or near-IR light irradiation. Molecules containing octahedral $\mathrm{Fe}$ (II) ions have been the most widely studied since the discovery of the light-induced excited spin-state trapping (LIESST) effect by Decurtins et al. [2,3] in [Fe(ptz) 6 (BF $\left._{4}\right)_{2}$ (ptz $=1$-propyltetrazole) which proved that the spin state could be switched reversibly from the low-spin (LS; $S=0$ ) to the high-spin (HS; $S=2$ ) state under visible light in a solid-state material. Since then $\left[\mathrm{Fe}(\mathrm{ptz})_{6}\right]\left(\mathrm{BF}_{4}\right)_{2}$ has become one of the archetypal examples of photoswitchable molecular complexes with an abrupt thermal HS-to-LS spin transition at $\sim 135 \mathrm{~K}$. At low temperature, green/blue light irradiation can switch the nonmagnetic LS Fe(II) ions to a metastable HS $S=2$ state, which has a long lifetime (several weeks at $2 \mathrm{~K}$ ). The LS ground state can be restored by thermal relaxation above the so-called $T_{L I E S S T}$ temperature $\left(T_{L I E S S T} \approx 55 \mathrm{~K}\right)$ or under irradiation using near-infrared light (reverse LIESST effect). Despite several studies of the photoinduced HS magnetic state, there is relatively little information regarding its magnetic anisotropy. From magnetization curves, Goujon et al. $[4,5]$ provided the first strong hint of the presence of a

\footnotetext{
*gregory.chaboussant@cea.fr
}

Published by the American Physical Society under the terms of the Creative Commons Attribution 4.0 International license. Further distribution of this work must maintain attribution to the author(s) and the published article's title, journal citation, and DOI. sizable zero-field splitting (ZFS). Using electron paramagnetic resonance (EPR) spectroscopy, Feng et al. [6] estimated both the axial $D$ and rhombic $E$ terms in the photoinduced high-symmetry HS state. Inelastic neutron scattering (INS) is a very efficient and proven technique to determine ZFS parameters [7], and unlike EPR, it does not rely on an external magnetic field to observe spin excitations. However, INS often requires a large amount of sample to reach reasonable counting times and statistics. It could thus be an extremely valuable tool for the study of photoswitchable magnetic molecular compounds, providing one succeeds in obtaining a significant fraction of HS magnetic sites over a large sample volume. So far, only small single crystals or thin layers of powder sample, up to $10-15 \mathrm{~mm}^{3}$, were considered in spectroscopic, diffraction, or bulk techniques due to light-penetration limits and laser-power damage. To best overcome this issue we have developed special setups tailored to combine neutron scattering (diffraction and INS) and in situ light irradiation for photomagnetic studies. The principal objective of the present study is to demonstrate the feasibility of such experiments with a large sample volume. We show that it is possible to determine by INS the ZFS parameters of the light-induced HS state of $\left[\mathrm{Fe}(\mathrm{ptz})_{6}\right]\left(\mathrm{BF}_{4}\right)_{2}$. Such a protocol could be easily implemented for other photomagnetic compounds.

\section{EXPERIMENTAL AND SAMPLE}

A fine powder of $\left[\mathrm{Fe}(\mathrm{ptz})_{6}\right]\left(\mathrm{BF}_{4}\right)_{2}$ was synthesized following the method developed by Feng et al. [6]. The powder neutron diffraction patterns were measured on the G4.1 diffractometer at LLB-Orphée (Commissariat à l'énergie atomique et aux énergies alternatives, Saclay), and the INS experiment was performed on the time-of-flight spectrometer FOCUS at the Swiss Spallation Neutron Source (SINQ, Paul Scherrer Institut, Villigen). An adapted sample holder designed to optimize the light irradiation over a large amount of powder sample was coupled to an optical fiber and fitted in the 
cryostat [8]. The sample is flash cooled in liquid nitrogen below the HS-to-LS spin transition temperature $(\approx 135 \mathrm{~K})$ so as to stay in the rhombohedral $R \overline{3}$ phase, thus avoiding a disordered phase $[9,10]$. The validity of such a procedure can easily be checked as the diffraction patterns of these two phases are completely different [11]. Several previous x-ray diffraction studies have investigated the crystal structure in the HS [high temperature (HT), 150-300 K] and LS [low temperature (LT), 10-90 K] states $[9,12]$. The lattice parameters of the photoexcited HS (LT) state at low temperature have also been reported. HS/LS fractions between $50 \%$ and $100 \%$ have been achieved under green light but only on tiny samples (volume $\sim 0.1-0.25 \mathrm{~mm}^{3}$ ) [9,13,14]. Green light sources $(510-530 \mathrm{~nm})$ were favored with the argument that choosing an excitation light energy slightly above the strong-absorption band, $\left({ }^{1} T_{1 g}\right) \leftarrow\left({ }^{1} A_{1 g}\right)$ (maximum at $\left.18400 \mathrm{~cm}^{-1}\right)$, will speed up the phototransformation. Another absorption band, $\left({ }^{1} T_{2 g}\right) \leftarrow\left({ }^{1} A_{1 g}\right)$ (maximum at $\left.26600 \mathrm{~cm}^{-1}\right)$, corresponds to blue/UV light. However, with blue light the process is more gradual, but the LIESST process remains very effective, as shown by Goujon et al. in polarized neutron diffraction (PND) experiments $[4,5]$. PND was used to map out the spin density of the photoinduced HS vs LS state at $2 \mathrm{~K}$ under $5 \mathrm{~T}$, with very encouraging results in terms of sample volume [15].

\section{NEUTRON DIFFRACTION}

The neutron diffraction data, obtained on the G4.1 diffractometer with a neutron wavelength $\lambda=2.423 \AA$ and a detector coverage $2 \theta=80^{\circ}$, are shown in Fig. 1(a). They have been fitted using the FULLPROF package [16] to the rhombohedral $R \overline{3}$ space group using the atomic positions previously determined because the resolution of the instrument did not allow us to refine the atomic positions reliably. We find $a, b=10.832(1) \AA$ and $c=31.850(2) \AA$ in the HS (HT, $140 \mathrm{~K})$ state and $a, b=10.657(2) \AA$ and $c=31.902(5) \AA$ in the LS (LT, $5 \mathrm{~K}$ ) state. Under light irradiation (405 and $450 \mathrm{~nm}$ ) we observe a progressive change in the Bragg peaks revealing the coexistence of the two spin states (HS and LS phases). During photoexcitation the temperature is stabilized at $10-15 \mathrm{~K}$, well below the temperature of the thermally-driven relaxation process. After photoexcitation and return to $5 \mathrm{~K}$ the lattice parameters are $a, b=10.829(5) \AA$ and $c=31.427(2) \AA$ for the HS phase, while those of the LS phase remain unchanged. Moreover, the Bragg peaks do not show any broadening or loss in intensity, suggesting that we stayed in the $R \overline{3}$ phase during the whole process. The obtained values are perfectly in agreement with previous $\mathrm{x}$-ray data, but extracting the HS/LS fraction from fitting the whole neutron diffraction pattern remains difficult. Instead, we chose to monitor the two Bragg peaks most sensitive to the Fe(II) spin state: (113) $\left(Q_{1} \approx 1.31 \AA^{-1}\right)$ and (202) ( $Q_{2} \approx 1.41 \AA^{-1}$ ). As shown in Fig. 1(a), under blue light the two peaks clearly evolve towards four peaks, one pair for each spin state; thus, they were fitted assuming two sets of Bragg peaks. The comparison of $Q_{1}$ and $Q_{2}$ Bragg peak intensities in both LS and HS states yields a reliable estimate of the HS fraction $\gamma_{H S}$ assuming $\gamma_{H S}=\frac{1}{2} \sum_{i=1,2} I_{Q_{i}}^{H S} /\left(I_{Q_{i}}^{H S}+I_{Q_{i}}^{L S}\right)$. The HS fraction $\gamma_{H S}$ as a function of time and illumination
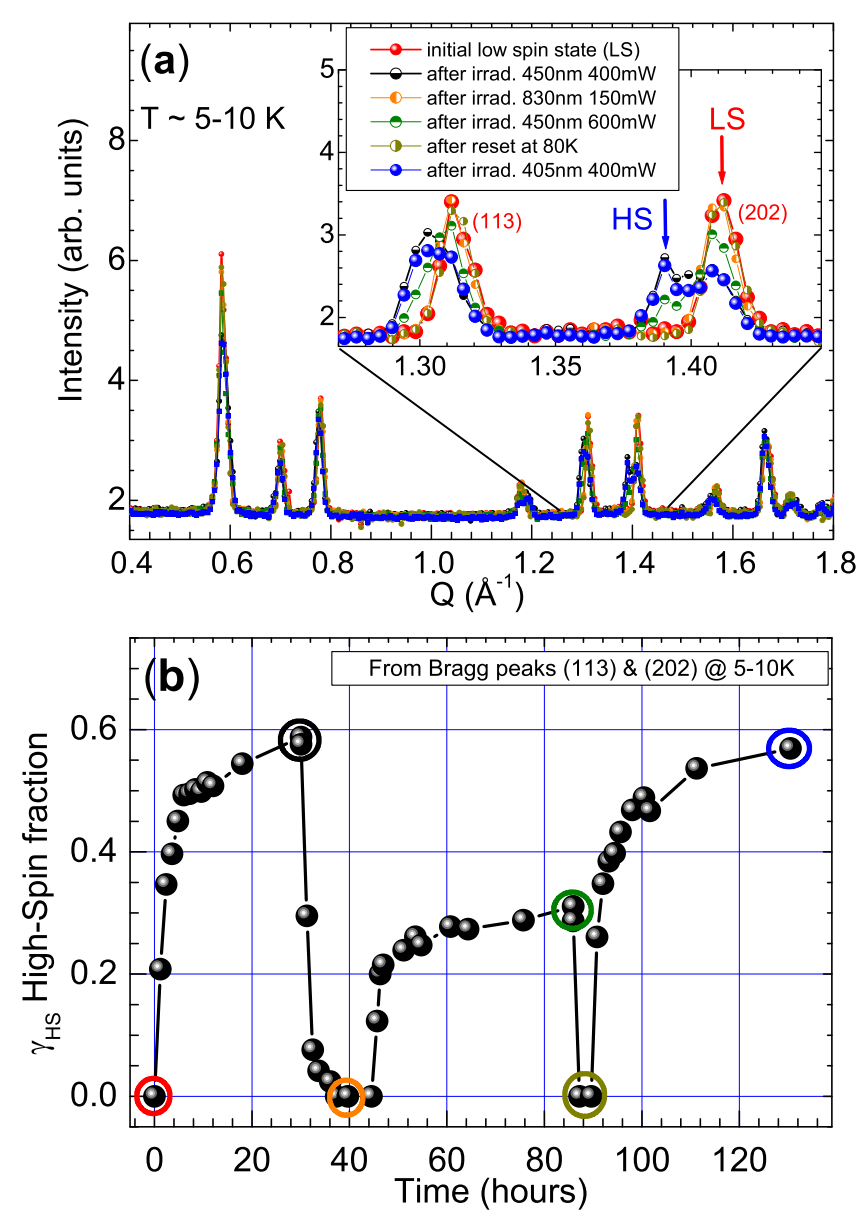

FIG. 1. (a) Powder neutron diffraction patterns at $T \approx 10 \mathrm{~K}$ of $\left[\mathrm{Fe}(\mathrm{ptz})_{6}\right]\left(\mathrm{BF}_{4}\right)_{2}$ under various light illumination conditions (without light: $T=5 \mathrm{~K}$ ). The inset focuses on the (113) and (202) Bragg peaks from which the HS fraction $\gamma_{H S}$ is derived from fits using Gaussian functions ( FWHM $=0.015(1) \AA^{-1}$ ). (b) High-spin fraction $\gamma_{H S}$ as a function of time and irradiation conditions, calculated from (113) and (202) Bragg peak intensities in both LS and HS states (see text). The colored circles refer to the selected diffraction patterns shown in (a). Red: after rapid cooling in liquid nitrogen to $5 \mathrm{~K}$ (100\% LS state); black: after irradiation at $\lambda=450 \mathrm{~nm}(400 \mathrm{~mW}, 32 \mathrm{~h})$; orange: after irradiation at $\lambda=830 \mathrm{~nm}(150 \mathrm{~mW}, 13 \mathrm{~h})$; green: after irradiation at $\lambda=450 \mathrm{~nm}(600 \mathrm{~mW}, 40 \mathrm{~h})$; dark yellow: $5 \mathrm{~K}$ after warming to $80 \mathrm{~K}$ to restore full LS state; blue: after irradiation at $\lambda=405 \mathrm{~nm}$ (400 mW, $40 \mathrm{~h})$.

conditions is shown in Fig. 1(b). The first LIESST effect using a 450-nm laser at $400 \mathrm{~mW}$ power leads to $\gamma_{H S} \approx 50 \%$ after 6-7 h, followed by a slower increase of $\gamma_{H S}$ up to $60 \%$. This crossover between fast and slow kinetics has been systematically observed in our experiments, and we attribute it to a complex thickness-dependent process associated with light penetration and diffusion inside the powder material [14]. The $450-\mathrm{nm}$ laser is then replaced by an $830-\mathrm{nm}$ laser (at $150 \mathrm{~mW}$ power), and a full reverse LIESST effect is observed after less than $5 \mathrm{~h}$. As the sample was kept below 10-15 K during light irradiation, this observed decay is undoubtedly due to the reverse LIESST process rather than a thermal relaxation effect. The second LIESST effect, still using a 450-nm laser but with more power $(600 \mathrm{~mW})$, is less efficient than the first 
$\left(\gamma_{H S} \approx 30 \%\right.$ at saturation), probably due to a locally higher sample temperature arising from excessive heating. During the 405-450-nm irradiation processes, the temperature close to the laser entry point may have been higher than on the outer parts of the sample, but the balance between photoconversion (LS to HS) and thermally activated relaxation (HS to LS) remains, however, positive, and the saturation at about $60 \%$ arises also partially from limited light penetration. At higher power, the LS-to-HS conversion process saturates at a lower level due to a higher local temperature. The level of HS fraction that is achieved appears to be limited by two processes: local temperature increase (but not severe enough to trigger a full conversion back to LS) and limited penetration of light inside the powder. There is thus probably a sizable amount of sample which does not experience light irradiation. This is supported by the fact that the coexistence of both LS and photoinduced HS phases is evidenced in the diffraction patterns. After having reset the whole sample to the LS state by first warming it up to $80 \mathrm{~K}$ and then returning it to $5 \mathrm{~K}$ [see Fig. 1(b)], we obtained a maximum similar HS/LS fraction (60\%) with 405-nm laser light $(400 \mathrm{~mW})$. With both laser wavelengths (405 and $450 \mathrm{~nm}$ ) we approach saturation for $\gamma_{H S}$ after $\sim 15 \mathrm{~h}$. In the course of the experiment, we did not reach a full $\mathrm{HS}$ state $\left(\gamma_{H S}=1\right)$, even after $30 \mathrm{~h}$. Despite only a fraction of the material being converted to HS, our neutron diffraction experiment demonstrates nevertheless that a large volume of powder sample (at least $175 \mathrm{mg}$ ) can be phototransformed using the present experimental setup. Such a positive outcome allowed us to perform INS experiments to probe the magnetic excitations of the light-induced metastable HS state as we expect no magnetic inelastic scattering at all in the LS phase.

\section{INELASTIC NEUTRON SCATTERING}

INS spectra were recorded on FOCUS at low temperature on $400 \mathrm{mg}$ of an undeuterated, microcrystalline powder sample of $\left[\mathrm{Fe}(\mathrm{ptz})_{6}\right]\left(\mathrm{BF}_{4}\right)_{2}$ placed in our adapted sample holder. The FOCUS data were measured with an incident neutron energy $E_{i}=9.108 \mathrm{meV}$ with instrumental FWHM $\approx 0.45 \mathrm{meV}$ at the elastic line position. The experimental resolution at finite-energy transfer is comparable (for $\hbar \omega \approx E_{i} / 2$, FWHM $\approx 0.6 \pm 0.1 \mathrm{meV}$; see [17]) with possible broadening due to multiple scattering arising from scattering of hydrogen nuclei. The data, corrected for detector efficiency with vanadium metal standard, correspond to the sum of all of the central detectors (the upper and lower banks of the detectors are obstructed by the bulky metallic cryomagnet). Figure 2 shows the evolution with magnetic field of the INS spectra at $10 \mathrm{~K}$ after laser light irradiation ( $405 \mathrm{~nm}, 400 \mathrm{~mW}, 100 \mathrm{~h})$. The INS spectrum obtained at $10 \mathrm{~K}$ and zero field prior to irradiation (100\% LS nonmagnetic state) has been subtracted from the three data sets. At zero field, after light irradiation, we observe a clear extra inelastic scattering signal at $\approx 4.0 \mathrm{meV}$, compared to the initial spectrum. Since the LS state is nonmagnetic, the main source of extra scattering is magnetic, although modifications in the low-energy phonon modes could be invoked. Under magnetic field (up to $6 \mathrm{~T}$ ) we observed a visible broadening and a slight shift towards higher energies of this extra scattering contribution, confirming its magnetic origin.
The ground state of the six-coordinated HS Fe(II) ions is the orbital triplet ${ }^{5} T_{2}$ state. The combined effect of spin-orbit coupling and additional ligand fields (e.g., tetragonal and/or rhombohedral distortions) has to be considered. These terms split the degeneracy of the ground term and quench, in many instances, the residual orbital contribution. Therefore, a "spin-only" Hamiltonian model has been conveniently, and successfully, used on various HS Fe(II) systems despite its approximative character (often taken care of in Landé factor shifts or "renormalization" of the anisotropy) [18-21]. There are several instances where the pertinence of the ZFS spin Hamiltonian has been discussed, even setting out the conditions where a spin-only Hamiltonian can or cannot be used to describe the low-energy states of Fe(II) in distorted octahedral symmetry [20,21]. In the present case, we have considered the model proposed by Feng et al. [6] used to describe their EPR data.

We assume that the six-coordinated Fe(II) ions in the HS state can be described using a $S=2$ spin Hamiltonian that includes both the ZFS term due to magnetocrystalline anisotropy and the Zeeman interaction term:

$$
\hat{\mathcal{H}}=\hat{S} \mathbf{D} \hat{S}+\mu_{B} \mathbf{B g} \hat{S} .
$$

D and $\mathbf{g}$ are the ZFS tensor and the $g$ matrix, respectively. $\hat{S}$ is the total spin moment of the molecule with components $\hat{S}_{i}$. In the proper coordinates, for which $\mathbf{D}$ is diagonal, one obtains

$$
\hat{S} \mathbf{D} \hat{S}=D\left(\hat{S}_{z}^{2}-\frac{S(S+1)}{3}\right)+E\left(\hat{S}_{x}^{2}-\hat{S}_{y}^{2}\right),
$$

where $D$ and $E$ are the axial and rhombic ZFS parameters, respectively. In the present case, the eventual presence of a rhombic term is not foreseen due to symmetry arguments unless some further distortions and/or local variation in crystallographic environment come into play (see [22] and references therein). Indeed, the $E$ term reported by Feng et al. [6] is rather small $(|E / D| \leqslant 0.06)$ and might be dominated by the existence of a random distribution of rhombic distortions. Introducing the reduced parameter $u=$ $\sqrt{3} E / D$, the zero-field eigenvalues are $E_{0}=-2 D \sqrt{1+u^{2}}$, $E_{1 a}=-D-3 E, E_{1 b}=-D+3 E, E_{2 a}=2 D$ and $E_{2 b}=$ $2 D \sqrt{1+u^{2}}$. For $D<0, E_{2 b}$ is the lowest-energy state. The corresponding eigenfunctions are detailed in Ref. [23]. In most cases we have $u^{2} \ll 1$, and the states $\psi_{2 a}$ and $\psi_{2 b}\left(M_{s}= \pm 2\right)$ are quasidegenerate $E_{2 a}=E_{2 b} \approx 2 D$. The next spin levels $\psi_{1 a}$ and $\psi_{1 b}\left(M_{s}= \pm 1\right)$ at $\hbar \omega=3|D|$ are split due to the possible rhombic term $E$. The energies of the allowed INS transitions $\Delta M= \pm 1$ between the low-lying states $\psi_{2 a, 2 b}$ and $\psi_{1 a}$ and $\psi_{1 b}$ are then $\hbar \omega_{ \pm} \approx 3|D| \pm 3|E|$. Finally, note that transitions between $\psi_{2 a, 2 b}$ and $\psi_{0}(\hbar \omega \approx 4|D|)$ are not allowed according to INS selection rules.

At zero field (see Fig. 2) we observe a broad INS peak centered around $3.9 \mathrm{meV}$, but its width is almost twice $(0.9 \pm 0.1$ $\mathrm{meV}$ ) the instrumental resolution, which suggests the presence of two peaks. The peaks shift slightly to higher energies with increasing field. Considering one single peak and keeping the resolution close to the instrumental one leads to a poor fit (not shown). If, instead, we use a double-Gaussian model with the same width as the resolution (or slightly larger) we obtain 

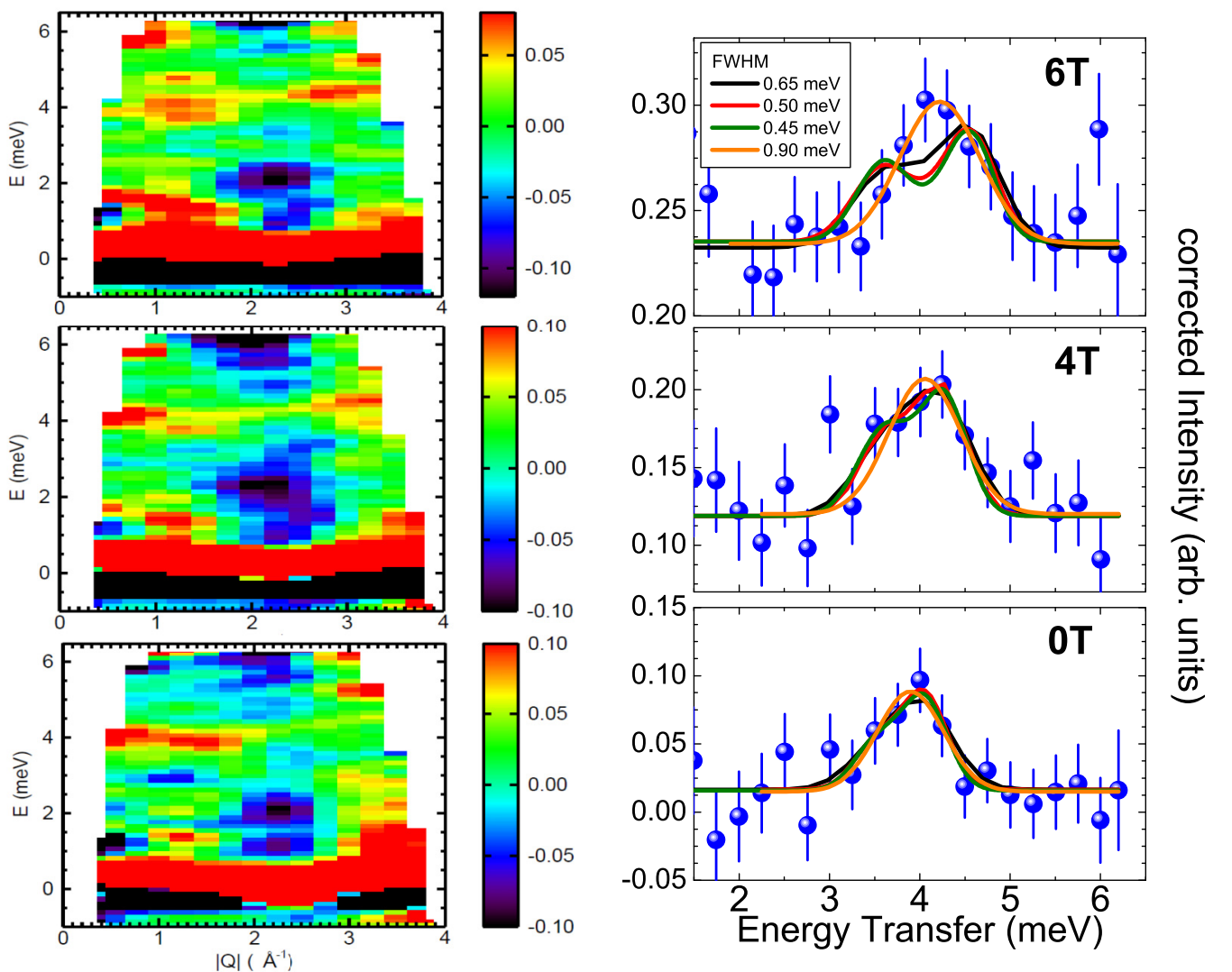

FIG. 2. Left: INS spectra at $10 \mathrm{~K}$ and different magnetic field values (from bottom to top: 0 , 4, and $6 \mathrm{~T}$ ) after laser light irradiation ( $405 \mathrm{~nm}, 400 \mathrm{~mW}$ ) for $\approx 100$ hours. The INS spectrum obtained at $10 \mathrm{~K}$ and zero field prior to irradiation (100\% LS nonmagnetic state) has been subtracted from the three data sets. The experiments were performed with $\lambda=3 \AA$, and the data are corrected with a vanadium standard. Right: One-dimensional cuts along energy transfer with an integrated $Q$ range of $0.6-3.0 \AA^{-1}$ and energy binning of 0.25 meV. Solid lines are best fits to the INS peaks (using Gaussian line shapes) derived from Eqs. (1) and (2) (see text). For the single-peak fit (orange line $), \mathrm{FWHM}=0.9( \pm 0.2) \mathrm{meV}$ and $\hbar \omega(0 T)=3.9 \pm 0.1 \mathrm{meV}, \hbar \omega(4 T)=4.06 \pm 0.1 \mathrm{meV}$, and $\hbar \omega(6 T)=4.2 \pm 0.1 \mathrm{meV}$. For double-peak fits, several energy resolutions (FWHM) were considered and kept fixed during the fits (see text): FWHM $=0.45$ (green line), 0.5 (red line), and $0.65 \mathrm{meV}$ (black line).

good agreement with two peaks centered at $3.6 \pm 0.1$ and $4.1 \pm 0.1 \mathrm{meV}$, but there is not enough resolution to clearly resolve the peaks. Taking into account these considerations, we tried to reproduce our INS data with ZFS parameters similar to those reported by EPR $(D<0$ and $E \neq 0)$ [6]. Identifying these INS peaks with the magnetic transitions of energies $\hbar \omega_{ \pm} \approx 3|D| \pm 3|E|$, we obtain $D=-1.28 \pm 0.03$ $\mathrm{meV}$ and $|E|=0.08 \pm 0.03 \mathrm{meV}$. The ratio $|E / D|=0.062$ is consistent with the initial assumption $\left(u^{2} \ll 1\right)$. As already shown by high-field (HF) EPR [6], the negative $D$ value signals the existence of an easy-axis-type anisotropy for the photoinduced HS Fe(II) ions. The parameters derived from INS are consistent with HF-EPR results ( $D$ is negative, with $|D| \leqslant 15 \mathrm{~cm}^{-1}=1.86 \mathrm{meV}$, and $E=-0.95 \mathrm{~cm}^{-1}=-0.12$ $\mathrm{meV}$ ) with reasonably small error bars (no error bars are provided from the EPR study).

With increasing magnetic field, the magnetic INS contribution undergoes a clear broadening and a small global shift towards higher energies. The energy shift, due to the Zeeman splitting of both $M_{s}= \pm 2$ and $M_{s}= \pm 1$ states, depends on the field orientation with respect to the easy-axis direction $\vec{z}$ (see Ref. [24]). In the parallel direction $\left(B_{z}\right)$, the Zeeman splitting of the $M_{s}= \pm 2$ states is $\delta_{z}^{\mid 2>}=\left[\Delta_{2}^{2}+\left(4 g_{z} \mu_{B} B_{z}\right)^{2}\right]^{1 / 2}$, where $\Delta_{2} \approx 3 E^{2} / D$ for small $E / D$. In such a situation, the overall splitting is essentially linear, $\delta_{z}^{\mid 2>} \approx 4 g_{z} \mu_{B} B_{z}$, down to $B_{z} \equiv$ $\Delta_{2} \approx 0.1 \mathrm{~T}$ without giving rise to an INS peak due to INS selection rules. At $10 \mathrm{~K}$ and $B_{z} \geqslant 4 \mathrm{~T}$, only the lowest Zeeman branch $\left(M_{s}=-2\right)$ will be thermally populated. On the other hand, the splitting of the $M_{s}= \pm 1$ states is given by $\delta_{z}^{\mid 1>}=\left[\Delta_{1}^{2}+\left(2 g_{z} \mu_{B} B_{z}\right)^{2}\right]^{1 / 2}$, where $\Delta_{1}=6 E \approx 3.8 \mathrm{~cm}^{-1}$ is comparable to the Zeeman term for $B_{z} \geqslant 4 \mathrm{~T}$. In the directions perpendicular to $\vec{z}$, the Zeeman interaction induces almost no additional splitting; it barely shifts the energies of the $M_{s}$ states by $\Delta E_{\perp}^{\mid 2>} \approx\left(g_{\perp} \mu_{B} B_{\perp}\right)^{2} / 3 D$ and $\Delta E_{\perp}^{\mid 1>} \approx$ $7\left(g_{\perp} \mu_{B} B_{\perp}\right)^{2} / 6 D$. Figure 2 shows a crude attempt to reproduce the observed INS peaks under magnetic field. Solid lines in the right panels are calculations derived from Eqs. (1) and (2), where we take $D=-1.28 \mathrm{meV},|E|=0.08 \mathrm{meV}$ (both inferred from zero-field estimates), and $g_{z} \approx 2.3$ (see [6]). From this set of data we certainly cannot unambiguously determine the $g$ factors. We therefore considered some reasonable values and tried to account for the field-dependence of the INS peak of our powder sample. Three energy resolutions $(0.45,0.50$, 
$0.65 \mathrm{meV}$ ) were considered to account for the combined effect of instrumental resolution and possible multiple scattering from hydrogen. In all cases, we find good agreement taking $g_{z}=2.3$ and $g_{x}=g_{y} \approx 1.6$. Note that the sensitivity to $g_{z}$ (controlled by the high-energy bound of the INS peaks) is higher than to $g_{x, y}$. This model is consistent with the observed broadening and the global shift of the INS peak under magnetic field. In particular, there is good agreement with the data at $B=0-4 \mathrm{~T}$, regardless of the resolution changes, but some discrepancy is visible for $B=6 \mathrm{~T}$, possibly due to magnetic torque effects that could substantially modify the statistical distribution of orientation in a powder.

\section{CONCLUSION}

In conclusion we have reported a neutron-scattering investigation of the photoinduced metastable HS $S=2$ state of $\left[\mathrm{Fe}(\mathrm{ptz})_{6}\right]\left(\mathrm{BF}_{4}\right)_{2}$. Using a specially designed setup, we demonstrated the feasibility to induce by blue light (405 and $450 \mathrm{~nm}$ ) irradiation a low-temperature spin transition (LIESST effect) over a large sample volume. In neutron diffraction, $\mathrm{HS} / \mathrm{LS}$ ratios of up to $60 \%(\approx 200 \mathrm{mg}$ of photoconverted material) are obtained during the LIESST effect, while a complete reverse LIESST is achieved using near-IR light $(830 \mathrm{~nm})$. The most important result is the INS study of the photoinduced HS state of a spin-crossover system. From
INS magnetic transitions, we have interpreted our data in terms of a spin-only Hamiltonian model to describe the metastable $S=2$ ground state and the presence of a zero-field splitting. Our scope was primarily to demonstrate that it is possible to perform inelastic neutron-scattering studies of photoinduced metastable states of photoswitchable materials. This result opens promising prospects for in situ magnetic inelastic neutron-scattering investigations of a broad range of photomagnetic materials.

\section{ACKNOWLEDGMENTS}

We are grateful to B. Gillon (CNRS) for useful discussions and to S. Ochsenbein (ETH Zürich) for help and advice during preliminary work at PSI. We acknowledge the technical support of T. Robillard (CEA) and S. Gautrot (CNRS). This work is supported by public grants overseen by the French National Research Agency (ANR) as part of the "Investissements d'Avenir" program [Labex NanoSaclay (Reference No. ANR-10-LABX-0035) and Labex PALM (Reference No. ANR-10-LABX-0039)]. This work is based on experiments performed at the Swiss spallation neutron source SINQ, Paul Scherrer Institute (Villigen, Switzerland), and at the Laboratoire Léon Brillouin (Gif sur Yvette, France). The UK Engineering and Physical Sciences Research Council is thanked for financial support (Grant No. EP/K033662/1).
[1] M. A. Halcrow, Spin-Crossover Materials: Properties and Applications (Wiley, Hoboken, NJ, 2013).

[2] S. Decurtins, P. Gütlich, C. P. Köhler, H. Spiering, and A. Hauser, Chem. Phys. Lett. 105, 1 (1984).

[3] S. Decurtins, P. Gütlich, K. M. Hasselbach, A. Hauser, and H. Spiering, Inorg. Chem. 24, 2174 (1985).

[4] A. Goujon, B. Gillon, A. Gukasov, J. Jeftic, Q. Nau, E. Codjovi, and F. Varret, Phys. Rev. B 67, 220401 (2003).

[5] A. Goujon, B. Gillon, A. Debede, A. Cousson, A. Gukasov, J. Jeftic, G. J. McIntyre, and F. Varret, Phys. Rev. B 73, 104413 (2006).

[6] X. Feng, C. Mathonière, I.-R. Jeon, M. Rouzières, A. Ozarowski, M. L. Aubrey, M. I. Gonzalez, R. Clérac, and J. R. Long, J. Am. Chem. Soc. 135, 15880 (2013).

[7] A. Furrer and O. Waldmann, Rev. Mod. Phys. 85, 367 (2013); R. Caciuffo, G. Amoretti, A. Murani, R. Sessoli, A. Caneschi, and D. Gatteschi, Phys. Rev. Lett. 81, 4744 (1998); I. Mirebeau, M. Hennion, H. Casalta, H. Andres, H. U. Güdel, A. V. Irodova, and A. Caneschi, ibid. 83, 628 (1999); R. Basler, C. Boskovic, G. Chaboussant, H.-U. Güdel, M. Murrie, S. T. Ochsenbein, and A. Sieber, Chem. Phys. Chem. 4, 910 (2003); A. Sieber, R. Bircher, O. Waldmann, G. Carver, G. Chaboussant, H. Mutka, and H.-U. Güdel, Angew. Chem., Int. Ed. 44, 4239 (2005); R. Bircher, G. Chaboussant, C. Dobe, H.-U. Güdel, S. T. Ochsenbein, A. Sieber, and O. Waldmann, Adv. Funct. Mater. 16, 209 (2006); K. Ridier, S. Petit, B. Gillon, G. Chaboussant, D. A. Safin, and Y. Garcia, Phys. Rev. B 90, 104407 (2014).

[8] The sample holder consists of two concentric 1-mm-thick quartz unpolished cylinders (inner diameters of 4 and $9 \mathrm{~mm}$, respectively, length of $54 \mathrm{~mm}$ ) fixed on one end to a quartz injector equipped with a plano-convex lens and at the other end to a quartz reflector. Light, inserted from a 0.5 -mm optical fiber, is transported across the cylinder and is scattered laterally to irradiate as much of the sample as possible. A $m=0.4 \mathrm{~g}$ fine-powder sample is placed in the void between the cylinders. The irradiated surface area is about $3000 \mathrm{~mm}^{2}$, and we estimate that the areal light power may be as high as $0.13 \mathrm{~mW} / \mathrm{mm}^{2}$ at a nominal source power of $P=400 \mathrm{~mW}$. The ensemble, once mounted, is then capped and kept in place by an aluminum socket sealed to a copper block. A similar setup is used in the diffraction experiment except that we use instead a single glass tube wrapped around the optic fiber with a $m=0.29 \mathrm{~g}$ powder sample placed around it. The irradiated surface area is about $430 \mathrm{~mm}^{2}\left(\approx 0.75 \mathrm{~mW} / \mathrm{mm}^{2}\right)$, with $P=400 \mathrm{~mW}$. In no instance did the sample suffer from light damage.

[9] J. Kusz, H. Spiering, and P. Gütlich, J. Appl. Crystallogr. 33, 201 (2000). Lattice parameters at $300 \mathrm{~K}$ (HS) were $a=$ $b=10.841 \AA, c=33.800 \AA$ and at $10 \mathrm{~K}(\mathrm{LS})$ were $a=b=$ $10.701 \AA, c=31.900 \AA$ ) without light. Lattice parameters in the excited HS state under green light $(514 \mathrm{~nm})$ were $a=b=10.882 \AA, c=31.480 \AA$.

[10] J. Kusz, P. Gütlich, and H. Spiering, Top. Curr. Chem. 234, 129 (2004).

[11] The diffraction patterns of the two possible low-temperature phases $(R \overline{3}$ and the disordered phase) are very different and cannot be mistaken. In particular, the most intense Bragg peak (003) at a low scattering angle experiences a large shift which signals immediately the nature of the phase.

[12] Y. Moritomo, K. Kato, A. Nakamoto, N. Kojima, E. Nishibori, M. Takata, and M. Sakata, J. Phys. Jpn. Soc. 71, 1015 (2002); For LS $(90 \mathrm{~K}) a=b=10.707 \AA, c=32.107 \AA$; for HS $(150 \mathrm{~K})$ $a=b=10.885 \AA, c=32.037 \AA$. 
[13] Y. Moritomo, K. Kato, A. Kuriki, A. Nakamoto, N. Kojima, M. Takata, and M. Sakata, J. Phys. Jpn. Soc. 71, 2609 (2002).

[14] Y. Moritomo, M. Kamiya, A. Nakamura, A. Nakamoto, and N. Kojima, Phys. Rev. B 73, 012103 (2006).

[15] J. Jeftic, B. Gillon, A. Goujon, Q. Nau, A. Gukasov, E. Codjovic, and F. Varret, Polyhedron 22, 2155 (2003). They achieved $\approx 80 \%$ HS fraction with $\lambda=532 \mathrm{~nm}\left(0.3 \mathrm{~mW} / \mathrm{mm}^{2}\right)$ on a sample volume of $4.8 \mathrm{~mm}^{3}$. Goujon et al. [4] achieved $\approx 100 \%$ HS fraction with $\lambda=473 \mathrm{~nm}\left(0.03 \mathrm{~mW} / \mathrm{mm}^{2}\right)$ on a sample volume of $15 \mathrm{~mm}^{3}$ (after $90 \mathrm{~min}$ of light exposure).

[16] J. Rodriguez-Carvajal, Physica B (Amsterdam, Neth.) 192, 55 (1993).

[17] J. Mesot, S. Janssen, L. Holitzner, and R. Hempelmann, J. Neutron Res. 3, 293 (1996); S. Janssen, J. Mesot, L. Holitzner, A. Furrer, and R. Hempelmann, Physica B (Amsterdam, Neth.) 234-236, 1174 (1997).
[18] Z. Ropka, R. Michalski, and R. J. Radwanski, Phys. Rev. B 63, 172404 (2001).

[19] A. L. Barra, A. K. Hassan, A. Janoschka, C. L. Schmidt, and V. Schunemann, Appl. Magn. Reson. 30, 385 (2006).

[20] G. Carver, C. Dobe, T. B. Jensen, P. L. W. Tregenna-Piggott, S. Janssen, E. Bill, G. J. McIntyre, and A.-L. Barra, Inorg. Chem. 45, 4695 (2006).

[21] A. Ferron, F. Delgado, and J. Fernandez-Rossier, New J. Phys. 17, 033020 (2015).

[22] R. Bircher, G. Chaboussant, A. Sieber, H.-U. Güdel, and H. Mutka, Phys. Rev. B 70, 212413 (2004).

[23] The eigenfunctions in the basis $\left|S, M_{S}\right\rangle$ are $\psi_{0}=\alpha^{-}(|+2\rangle+$ $|-2\rangle) \sqrt{2}-\alpha^{+}|0\rangle, \quad \psi_{1 a}=(|+1\rangle-|-1\rangle) \sqrt{2}, \quad \psi_{1 b}=(|+1\rangle+$ $|-1\rangle) \sqrt{2}, \quad \psi_{2 a}=(|+2\rangle-|-2\rangle) \sqrt{2}, \quad \psi_{2 b}=\alpha^{+}(|+2\rangle+$ $|-2\rangle) \sqrt{2}+\alpha^{-}|0\rangle$. The parameter $\alpha^{ \pm}$is defined as $\alpha^{ \pm}=\sqrt{\frac{1}{2}\left[1 \pm\left[1+(E / D)^{2}\right]^{-1 / 2}\right]}$.

[24] R. Boča, Theoretical Foundations of Molecular Magnetism (Elsevier, Lausanne, 1999). 